\title{
Influence of Nonoperative Treatments for Subacromial Shoulder Pain: A Review Article
}

\author{
Tengku Fadilah Tengku Kamalden ${ }^{1, *}$, Qais Gasibat ${ }^{1}$, Agiela Emhemed Rafieda ${ }^{2}$, \\ Walied Ashoor Sulayman ${ }^{3}$, Roxana Dev Omar Dev ${ }^{1}$, Alif Syamim Syazwan ${ }^{1}$, Mohd Rozilee Wazir ${ }^{1}$ \\ ${ }^{1}$ Department of Sport Studies, University Putra Malaysia (UPM), 43000 Serdang, Selangor, Malaysia \\ ${ }^{2}$ Department of Physiotherapy, Faculty of Medical Technology, Misurata, Libya \\ ${ }^{3}$ University of Mediterranean Karpasia, Şht. M.Ruso Cad. No:79 Küçükkaymaklı, Nicosia, Cyprus
}

Received November 29, 2020; Revised January 26, 2021; Accepted February 17, 2021

\section{Cite This Paper in the following Citation Styles}

(a): [1] Tengku Fadilah Tengku Kamalden, Qais Gasibat, Agiela Emhemed Rafieda, Walied Ashoor Sulayman, Roxana Dev Omar Dev, Alif Syamim Syazwan, Mohd Rozilee Wazir, "Influence of Nonoperative Treatments for Subacromial Shoulder Pain: A Review Article," International Journal of Human Movement and Sports Sciences, Vol. 9, No. 2, pp. 275 - 293, 2021. DOI: 10.13189/saj.2021.090216.

(b): Tengku Fadilah Tengku Kamalden, Qais Gasibat, Agiela Emhemed Rafieda, Walied Ashoor Sulayman, Roxana Dev Omar Dev, Alif Syamim Syazwan, Mohd Rozilee Wazir (2021). Influence of Nonoperative Treatments for Subacromial Shoulder Pain: A Review Article. International Journal of Human Movement and Sports Sciences, 9(2), 275 - 293. DOI: 10.13189/saj.2021.090216.

Copyright $\odot 2021$ by authors, all rights reserved. Authors agree that this article remains permanently open access under the terms of the Creative Commons Attribution License 4.0 International License

\begin{abstract}
Sub-acromial pain syndrome (SAPS) in overhead athletes is the most common reason for shoulder pain. As a rational decision, the medical treatments of today will likely be the best cure for this particular population. Conservative techniques are generally utilised to treat inflammation and pain through electrophysical approaches or to control the subacromial space to avoid impingement by rotator cuff and scapular muscle exercises, as the SAPS (subacromial pressure) mechanism remains unclear. The aim of this analysis is to assess the efficiency of these measures for pain reduction and function improvement. The literature, the best research source to be used, was searched using such search engines as PubMed, Medline, and Google Scholar. The articles were chosen in a manner that relates to the study objective and also their scientific relevance. This research may inform that in subjects with a reduced subacromial space, exercise can have a greater impact on overhead athletes. The effectiveness of centred therapy with Extracorporeal Shockwave (ESWT) on severe plantar fasciitis is uncertain. A greater cohort with its training efficiency in comparison with the conventional physiotherapy programme is required to demonstrate the efficacy of SAPS bodyblade exercise on overhead athletes.
\end{abstract}

Keywords Conservative Intervention, Exercises, Electrophysical, Bodyblade Exercise, Subacromial Pain
Syndrome, Extracorporeal Shockwave

\section{Introduction}

A common shoulder disorder found in sports medicine is Subacromial Pain Syndrome (SAPS) [28]. Among the treatments provided for non-operational areas, including physical exercise, modalities of electrotherapy that help alleviate pain and improve function, the methods are varied, and include a wide range of options. In order to normalise scapular and glenohumeric activity, certain fitness programmes consist of calming, regional and global reinforcement exercises [11], while the focus of extracorporeal shockwave (ESWT) therapy is on the supraspinate tendon in order to produce positive physiological effects. Although it has already been shown that exercise has a beneficial effect on pain and function after intervention, the intermediate effects of treatment and ESWT, as well as their mechanisms, are still unclear. Physiotherapists have been using electrophysical therapy for relief of pain in the shoulder such as pulsed electric field, laser and ultrasound [1,2].

Extracorporeal Shockwave Treatment (ESWT) is a fairly recent approach that is used to treat this disorder. ESWT has been successfully applied to a wide variety of 
musculoskeletal disorders, such as pain in the shoulder, elbow, knee and ankle. Traditionally, there are two types of threnody blows, namely the centred and the radial. A high peak pulse wave with a pulse width of $1 \mu$ s (equal to $1 \mathrm{MHz}$ ) and a peak pressure above 50 bar is the fundamental ESWT [18].

SAPS is related to tightness of the soft tissue [36] and muscle fatigue [35]. This dysfunction can affect the subacromial space and cause the underlying structures, such as the supraspinatus tendon, to be overly compressed [37]. The standard exercise programme consists of relaxing the soft tissue and positive reinforcement exercises have been reported to reduce the pain $[32,40]$ and function $[16,32,37,48,52]$ at week 4 through week 24 . However, in these above-mentioned studies, the participants were middle-aged individuals (mean age ranged from 44 to 58), and the level of activity was not recorded. In younger overhead athletes, disease-induced pathological changes can differ from those of 100 middle-aged individuals. Ludewing et al. [32] found that the feature shift was however, below the minimum clinically observable difference in a group of middle-aged (mean age 49) construction workers. More recent studies have indicated exercise style and intensity have an effect on their functional outcomes [50].

An article written in Nature [24] stated that exercise that focuses on the shoulder muscle area, and combined with manual therapy, is more effective than exercise that does not focus on the muscles in the shoulder area. Studies have shown that high-intensity exercises that require from upper to super-classification levels of exercise have shown greater benefits than a moderate intensity category [40]. It is believed that when throwing an object (such as a baseball) a piece of the energy from the lower part of our bodies is changed into the upper part of our bodies. The energy then goes to the chest and goes through the heart. This gets changed into the shoulder, and as it makes it way to the arm, the arm then completes the last link to the peak. Any mild weakness in the lower extremity or trunk regions areas of the body may affect the movement of the shoulder [45].

Popularised in the 70 's, Bodyblade fitness is a modern form of exercise that involves trunk and shoulder muscle mobilisation by swinging a flexible blade at 270 vibrations per minute [30]. One of the ways of helping an aching patient heal is to use a type of treatment called kinesio taping, in which taping is applied to someone's body. After this is done, the injured area feels better [29]. When these studies were done using the Bodyblade exercise, the results showed that it could be an alternative solution to traditional exercise. The details concerning the intermediate implications of this research have not yet been examined in overhead athletes. After an athlete sustained a glenohumeral dislocation injury, significant changes in their pain and joint function were recorded. The bodyblade allows for a highly intense physical activity involving trunk and shoulder muscle mobilisation, thus shaking a lightweight blade at 270 vibrations per minute. The mechanism of treatment is suggested as being a decline in neuromuscular development, decline in the joint proprioception, and decline in accuracy in the movement [30]. As a result of all this, the bodyblade exercise can be used in a different way or for different patients other than traditional exercises.

Comparable to incisions and punch through for the shoulder pain of SAPS, ESWT has been utilised to treat shoulder pain $[17,43]$.

Recent research by Galasso et al. [17] has shown that regulation therapy using electrical stimulation of the foot is more effective and leads to improvement in movement and recovery in three months. It is known that one of the mechanisms of the action of ESWT includes regulating the blood flow in the tendon, and facilitating tendon repair [39]. Keenly noted, the reduced vascularity of the tendon supraspinatus was, in fact, immediate after application of ESWT in case-series studies, followed by reduced pain terms in the first two weeks post procedure [38]. Should similar findings be found in three months post prophylactic, this would be worth looking into. After conducting a three-month controlled trial test on ESWT and Bodyblade, it was found that both of these therapies are very helpful in decreasing the deficits in understanding the effects of traditional exercise in the area of motivation for pain control and function.

By analysing the changes in the subacromial space and the response of the tendon to specific medication, it is possible to deduce what the relationship is between that and the response of the actual healing symptoms of the patient.

There are a lot of people, who are addicted to exercise, that have SAPS. The idea behind the concept is based on the theory of impingement-instability that excessive superior migration of the humeral head causes the subacromial space to narrow. This can result in impingement of the subacromial tissues, resulting in pain and dysfunction. The rotator cuff muscle is responsible for driving the humeral head. With the hand and the elbow in alignment, the elbow stabilises the humeral head so the lower arm may be pushed forward. The supraspinatus muscles also serve to keep the upper arm under the bone (vena cava) sagging. Research shows that this prevents excessive arm motion [14]. In contrast, the shoulder rotators on the thoracic side provide the scapular with a synchronous rotation during the humeral movement and a stable base from which to activate the rotator cuff [28]. In addition, this study suggests that reduced internal rotation of the shoulder as a measurement of the tightness of the capsule can contribute to anterior and higher migration of the humeral head [23].

In response, shoulder strengthening exercises are performed to maximise glenohumeral motion, improved scapular and rotator cuff strength and improved muscular activation. This can be achieved with internal rotation exercises to optimise scapular depression. The purpose of 
this research is to evaluate the effects of the relief of pain and improvement of the functions of conventional exercise, ESWT, Bodyblade exercise in SAPS.

\section{Method}

The following keywords and variations of these terms were searched for relevant keywords in work-related electronic databases, including PubMed, ScienceDirect, Protection Article Archive, and Google Scholar: Conservative Interference, exercises, Electrophysical, Bodyblade Exercise, Subacromial Pain Syndrome, Extracorporeal Shockwave.

\section{Results}

A total of twenty-two trials were performed in conjunction with monitoring or other treatments in patients with SAPS with exercise therapy, and four clinical studies compared control group exercise, three of which demonstrated positive outcomes for pain relief or control group exercise task improvement $[7,31,32]$. When taking a measure of pain magnitude, the pain was reduced in 2 to 3 out 10 of the Visual Analogue Scale or $10.8 \%$ of the Impairment for Shoulder Hand Score (DASH) [31]. There was a modest but consistent rise in the improvement rate over the 2 to 3 years of the study, from $60 \%$ to $68 \%$. At first, the average speed of the growth was around 2 weeks a month; then, it changed to 4 weeks in a month; then, it went slower than that. The most recent analyses of the impact, forms, and strength of supervision on pain management and work revealed three significant themes. High-dose workouts and precise exercises have been found to relieve discomfort and improve function better than low-dose or non-specific exercises [40]. Though it is not proven scientifically, the increased quality of life caused by exercising almost every day is recorded and proven. Twelve researchers went through the rigors of measuring the difference of exercise, diet, and medication. Physical exercises are much more common than electric procedures such as ESWT, which is far more popular than ESWT $[15,16]$. Exercise therapy has been found to have similar effects such as surgery $[7,21,41]$, injections [9,19], and functional braces [46]. One aim of the study was to compare the effects of combined physical activities (exercise) with the effects of physical activity alone. There has been a wide range of such contradictory studies concerning the relationship between manual therapy and exercise. For example, an extensive follow-up study outlined by Bang et al. [2] and Kachingwe et al. [25] demonstrated that those with more muscle strength experienced a much larger increase in strength than the rest. The results of studies $[3,44,48]$ suggest the treatment decisions are not very different between the two options. Ginn et al. [19] observed this same effect in their study where the mixed and exercise groups spent equal amounts of time exercising physically. Particularly with the use of exercise, the results are very promising with 61 to $69 \%$ of people in pain showing improvements in their functionality, and patients being able to tolerate the therapy better. Furthermore, it has the same psychological or psychological disruption effect of a surgical operation or an injection. Two clinical trials compared the effect of ESWT with placebo ESWT on non-calcific shoulder tendinosis. Schmitt et al. found that there was no significant difference in pain relief between ESWT and control groups with an amplitude of $0.11 \mathrm{~mJ} / \mathrm{mm} 2$ for 2,000 shocks in three sessions. Of fifty per cent of the participants, only about half would recover in the recovery group [43].

It was found by Galasso et al. [17] that positive results have been found for ESWT-based deep tissue therapy three month post-intervention over power. It found that the effectiveness of the program was $63.7 \%$ of the patients for three months post intervention. The investigators were pleased with some of the children cared for by making follow-ups calls nine years after the intervention. During the study, the authors used a comparatively limited dosage of $0.068 \mathrm{~mJ} / \mathrm{mm} 2$ and gave more of it, in larger doses, to test animals while testing. Based on the studies, the effectiveness of the specific focal electro-acupuncture was not yet known. As a result of the difference in dose between the equipment and the drug used, there was an increase in the variance in the sample. Notarnicola et al. [38] reviewed the literature and discovered that ESWT (ESWT focused on pain and oxygen tissue relief) could be a useful treatment for individuals with supraspinatus tendinosis. The investigators administered 2,000 impulses of 0.04 to 0.07 $\mathrm{mJ} / \mathrm{mm} 2$ over the course of the study, and found considerable pain reduction in $65.6 \%$ of the testing participants, as well as a drop in oxygen tissue saturation that occurred after the usage of focused ESWT exams on patients. Lack of pain reduction with the use of this method was linked to a problem with the oxygen levels of some individuals [51].

Due to this lack of research, no one knows for sure if the routine exercise using the Bodyblade is physically sound. The article on glenohumeral dislocations in a journal [8] describes a case study where the researchers documented pain relief for a patient with a glenohumeral dislocation. The therapy method involving elastic bands and a body blade workout showed a demonstrable stabilising impact on nine ice hockey athletes suffering from shoulder weakness [29]. In order to have proper training for the Bodyblade exercise with the SAPS type of athletes, a greater training group is required made up of athletes that have a greater variety of and in greater in difficulties compared to the other types of programme. Table 1 to Table 8 illustrates the effectiveness of the various therapies used in pain treatment illustrated in improvements in terms of pain and function. 
Table 1. Comparison of Exercise vs Control for SAPS

\begin{tabular}{|c|c|c|c|c|c|c|c|}
\hline Author & Participants & Age & Intervention & Frequency & Follow up & Outcome measures & Results \\
\hline $\begin{array}{c}\text { Brox et al. } \\
\text { [7] }\end{array}$ & $\begin{array}{c}\mathrm{N}=125 \text { (Male: } 54 ; \\
\text { Female: } 71 \text { ) }\end{array}$ & 18 to 66 & $\begin{array}{l}\text { 1.Arthroscopic surgery }(\mathrm{n}=31) \\
\text { 2. Placebo laser }(\mathrm{n}=13) \\
\text { 3. Supervised exercise }(\mathrm{n}=33) \\
\text { Sling suspension exercises, wall } \\
\text { push-ups, pulley exercises and light } \\
\text { elastic band internal rotation, external } \\
\text { rotation in standing for } 1 \text { hour daily }\end{array}$ & $\begin{array}{c}12 \text { sessions for } 6 \\
\text { weeks in placebo } \\
\text { group } \\
\text { 2/weeks plus home } \\
\text { exercise programme } \\
\text { for } 3 \text { to } 6 \text { months in } \\
\text { an exercise group }\end{array}$ & 3,6 months, 2.5 years & $\begin{array}{l}\text { Pain: Numeric pain } \\
\text { rating scale } \\
\\
\text { Function: Neer } \\
\text { shoulder score Scores } \\
\text { for pain and disability } \\
\text { were dichotomised and } \\
\text { considered successful } \\
\text { if scores before } \\
\text { randomisation were } \\
\text { reduced }>50 \% \text { at } \\
\text { follow-up }\end{array}$ & $\begin{array}{l}6 \text { months: The success rate of both } \\
\text { exercise }(61.4 \%) \text { and surgery } \\
(68.4 \%) \text { groups were significantly } \\
\text { better than the placebo group }(25 \%)\end{array}$ \\
\hline $\begin{array}{l}\text { Lombardi } \\
\text { et al. [31] }\end{array}$ & $\begin{array}{l}\text { N=60 (Male: 14; } \\
\text { Female: } 46 \text { ) }\end{array}$ & $\begin{array}{c}\text { Group 1: } \\
54.8 \pm 9.4 \\
\\
\text { Group 2: } \\
56.3 \pm \\
11.1\end{array}$ & $\begin{array}{l}\text { 1. Exercise group }(\mathrm{n}=30) \text { Resisted } \\
\text { flexion/extension/ internal rotation, } \\
\text { external rotation, } 1 \text { set of } 8 \text { reps at } \\
50 \% \text { of the } 6 \text { repetition maximum } \\
\text { followed by } 2 \text { minutes rest and } 1 \text { set of } \\
70 \% \text { of the } 6 \text { repetition maximum, } \\
\text { Speed of the movement } 2 \text { seconds for } \\
\text { the concentric and eccentric phase. } \\
\text { Multi-pulley equipment used for } \\
\text { exercises. } \\
\text { 2. Control }(n=30)\end{array}$ & $\begin{array}{l}\text { Visual analogue } \\
\text { scale }\end{array}$ & $\begin{array}{l}\text { Pain: } 10 \mathrm{~cm} \text { visual } \\
\text { analogue scale } \\
\text { Function: DASH } \\
\text { quality of life: } \\
\\
\text { Short Form } 36 \text { range of } \\
\text { motion } \\
\\
\text { Strength: Isokinetic } \\
\text { assessment, measured } \\
\text { in } 3 \text { planes of } \\
\text { movement at } 600 / \mathrm{s} \\
\text { and } 1800 / \mathrm{s} \text {. }\end{array}$ & & $\begin{array}{l}\text { As compared with the control group, } \\
\text { the exercise group showed a } \\
\text { statistically significant decrease in } \\
\text { pain relief and impairment. } \\
\text { Discomfort decreased from } 4.2 \mathrm{~cm} \\
\text { to } 2.4 \mathrm{~cm} \text { at rest and from } 7.4 \text { to } 5.2 \\
\mathrm{~cm} \text { during activity. DASH decreased } \\
\text { from } 44.0 \text { to } 33.2 \text { in the exercise } \\
\text { category. }\end{array}$ \\
\hline $\begin{array}{l}\text { Kachingwe } \\
\text { et al. [25] }\end{array}$ & $\begin{array}{l}\mathrm{N}=33 \text { (Male }: 17 \\
\text { Female }: 16 \text { ) }\end{array}$ & 18 to 74 & $\begin{array}{l}\text { 1. Supervised exercise only: posterior } \\
\text { capsular stretching, posture correction } \\
\text { exercises, rotation of the cuff (external } \\
\text { rotation of the elastic resistance), } \\
\text { scapular stability exercise (elastic } \\
\text { resistance exercises), ice pack } \\
\text { 2. Supervised exercise as per group } 1 \\
\text { with glenohumeral joint mobilisations } \\
\text { 3. Supervised exercises as per group } 1 \\
\text { with Mulligan mobilisations } \\
\text { technique. } \\
\text { 4. Control group with advice only. }\end{array}$ & $\begin{array}{c}\text { Group } 1 \text { to } 3 \text { received } \\
\text { Physiotherapy } \\
\text { x1/week x } 6 \text { weeks } \\
\text { with home exercise } \\
\text { programme daily }\end{array}$ & 6 weeks & $\begin{array}{l}\text { Pain: } 10 \text { point visual } \\
\text { analogue scale } \\
\text { range of motion: } \\
\text { Goniometric } \\
\text { measurement of flex } \\
\text { and scaption } \\
\text { Function: shoulder } \\
\text { pain and disability } \\
\text { index }\end{array}$ & $\begin{array}{c}\text { Repeated analyses of treatments } \\
\text { demonstrated substantial decreases } \\
\text { in pain, enhanced performance and } \\
\text { increased active range of movement } \\
\text { for each dependent variable, } \\
\text { univariate analyses of pre-post } \\
\text { treatment progress showed no } \\
\text { statistically significant differences } \\
\text { between the four groups. } \\
\text { Exercise category vs Control: visual } \\
\text { analogue scale: } 14.4 \text { (119.8) \% vs } \\
20.8(112.3) \% \text { Neer: } 46.4(49.5) \% \\
\text { vs } 44.0 \text { (57.2) \%: } 11.2(130.7) \% \text { vs } \\
39.5(54.9) \% \text { shoulder pain and } \\
\text { disability index } \\
: 34.2(58.9) \% \text { vs } 61.6 \text { (35.9) \% }\end{array}$ \\
\hline
\end{tabular}


Table 1 Continued

\begin{tabular}{|c|c|c|c|c|c|c|c|}
\hline $\begin{array}{l}\text { Ludewig et } \\
\text { al. [32] }\end{array}$ & $\begin{array}{l}\mathrm{N}=92 \text { (overhead } \\
\text { male construction } \\
\text { workers) }\end{array}$ & $\begin{array}{c}\text { Mean age } \\
49\end{array}$ & $\begin{array}{l}\text { 1. Exercise group } \\
\text { Stretches: pectoral minor and } \\
\text { posterior shoulder stretches }(2 \times 30 \mathrm{~s} \text {, } \\
5 \mathrm{x} / \mathrm{day}) \text {; upper trapezius muscle } \\
\text { relaxation }(5 \mathrm{x} / \mathrm{day}) \text {; and strengthening } \\
\text { of anterior and humeral serratus } \\
\text { external rotation (progress from } 3 \times 10 \\
\text { to } 3 \times 20 \text { reps, } 3 \text { days/week) } \\
\text { 2. Control ( } \mathrm{n}=33) \\
\text { 3. Asymptomatic control }(\mathrm{n}=25)\end{array}$ & $3 \mathrm{x} /$ week for 8 weeks & $\begin{array}{l}8 \text { to } 12 \text { weeks post } \\
\text { treatment }\end{array}$ & $\begin{array}{l}\text { Pain and function: } \\
\text { i) Shoulder rating } \\
\text { questionnaire. ii) } \\
\text { Modified shoulder } \\
\text { pain and disability } \\
\text { index }\end{array}$ & $\begin{array}{c}\text { Shoulder rating questionnaire and } \\
\text { shoulder satisfaction score } 6.2(0.35) \\
\text { vs } 5.0 \text { (3.72) percent (group } 2) \& \\
0.36 \text { (1.65) (group 3) showed } \\
\text { statistically significantly greater } \\
\text { changes than control groups. } \\
\text { Shift in per cent of shoulder rating } \\
\text { questionnaire } \\
\text { Group } 1 \text { vs Group } 2 \text { vs Group } 3: \\
19.23 \text { (4.75) } \% \text { vs }-0.27(3.72) \% \text { vs } \\
0.36161(1.65) \% \text { Pre-post test score } \\
\text { of satisfaction score } \\
\text { Group } 1 \text { vs Group } 2 \text { vs Group } 3: \\
4.5(0.31) \text { to } 6.2(0.35) \text { vs } 5.0(0.31) \\
\text { to } 5.0(0.34) \text { vs } 9.1(0.37) \text { to } 8.8 \\
(0.40)\end{array}$ \\
\hline
\end{tabular}


Table 2. Comparison of High Dosage with Low Dosage Exercise or Supervision of Exercise

\begin{tabular}{|c|c|c|c|c|c|c|c|}
\hline Author & Participants & Age & Intervention & Frequency & Follow up & $\begin{array}{l}\text { Outcome } \\
\text { measures }\end{array}$ & Results \\
\hline $\begin{array}{c}\text { Granviken et al. } \\
\text { [20] }\end{array}$ & $\mathrm{N}=46$ & 18 to 65 & $\begin{array}{l}\text { 1. Home exercise group ( } \mathrm{n}=23) \text { : } \\
\text { One Supervised treatment +home } \\
\text { exercises } \\
\begin{array}{l}\text { 2. Supervised exercise group } \\
(\mathrm{n}=23): 10 \text { supervised treatments } \\
\text { +home exercises }\end{array}\end{array}$ & $\begin{array}{l}6 \text { weeks intervention including } \\
\text { scapular stabilising exercises, } \\
\text { rotator cuff exercises and } \\
\text { pain-free range of motion } \\
\text { exercises; } 30 x 3 \text { sets performed at } \\
\text { home, } 4 \text { to } 6 \text { exercises } 2 / \text { day } \\
\text { everyday }\end{array}$ & $\begin{array}{c}\text { 6th and } \\
\text { 26th week }\end{array}$ & $\begin{array}{l}\text { Shoulder pain and } \\
\text { disability index } \\
\text { visual analogue } \\
\text { scale: numeric } \\
\text { pain rating scale }\end{array}$ & $\begin{array}{l}\text { Both groups changed substantially, with } \\
\text { no major variations in the shoulder pain } \\
\text { and disability index at } 6 \text { weeks ( } 0 \text { points, } \\
95 \text { percent CI-14 to } 14 \text { ) or } 26 \text { weeks } \\
\text { follow-up ( }-2 \text { points, } 95 \text { percent CI- } 21 \text { to } \\
\text { 17) There were no differences in pain } \\
\text { between groups at either time }>=2 \\
\text { positive tests Home exercises vs } \\
\text { supervised exercises: } 85.71 \text { percent vs } \\
47.83 \text { percent. }\end{array}$ \\
\hline Holmgren et al. [24] & $\mathrm{N}=97$ & 30 to 65 & $\begin{array}{l}\text { 1. Specific exercise group }(\mathrm{n}=51) \text { : } \\
\text { Two eccentric exercises for rotator } \\
\text { cuff, three concentric/eccentric } \\
\text { exercises for the scapular stabilisers } \\
\text { and posterior stretch } \\
\text { 2. Control exercise group ( } \mathrm{n}=46) \text { : six } \\
\text { unspecific movement exercises for } \\
\text { neck and shoulder without external } \\
\text { load }\end{array}$ & $\begin{array}{l}\text { Group 1: } 15 \times 3 \text { sets twice daily } \\
\text { for } 8 \text { weeks; } 30 \text { to } 60 \text { seconds of } \\
\text { posterior stretch, repeated three } \\
\text { times twice daily for } 8 \text { weeks; } \\
\text { once a day from week } 8 \text { to } 12 \text {. } \\
\text { Group 2: Repeated } 10 \text { times for } \\
\text { each movement exercise and } \\
\text { three times twice daily at home } \\
\text { for each stretching exercise }\end{array}$ & 3 months & $\begin{array}{l}\text { Constant-Murley } \\
\text { Score DASH } \\
\text { Successful } \\
\text { outcome (defined } \\
\text { as large } \\
\text { improvement or } \\
\text { recovered) }\end{array}$ & $\begin{array}{l}\text { Group } 1 \text { vs Group 2: Constant-Murley } \\
\text { score score: } 24 \text { vs } 9 \text { points (significant) } \\
\text { Successful outcome: } 69 \% \text { vs } 24 \%\end{array}$ \\
\hline Osteras et al. [40] & $\begin{array}{c}\mathrm{N}=61 \\
\text { (Male: } 10 \\
\text { Female : } 51 \text { ) }\end{array}$ & 18 to 60 & $\begin{array}{l}\text { 1. High-dosage exercise: } 11 \\
\text { exercises, total of } 36 \text { treatments } \\
\text { ( } 3 \times 30 \text { repsx3/weeksx3 month) } \\
\text { including } 35 \text { to } 40 \text { minutes static } \\
\text { cycling at moderate to high } \\
\text { intensity, } 70 \text { to } 80 \% \text { maximum heart } \\
\text { rate } \\
\text { Resisted shoulder flexion (deloaded } \\
\text { pulley exercise), resisted extension } \\
\text { (dumbbells), elbow } \\
\text { flexion/extension (dumbbells), } \\
\text { external rotation, internal rotation, } \\
\text { abduction (deloaded shoulder } \\
\text { pulley). } \\
\text { 2. Low-dosage exercise: } 6 \text { exercises } \\
\text { (2x10 reps each exercise) plus } 5 \text { to } \\
10 \text { minutes of static cycling at } \\
\text { moderate to high, } 70 \text { to } 80 \% \\
\text { maximum heart rate. }\end{array}$ & $\begin{array}{l}\text { X3 physiotherapy sessions/ week } \\
\text { x } 12 \text { weeks }\end{array}$ & 3 months & $\begin{array}{l}\text { Pain: } 100 \text { visual } \\
\text { analogue scale. } \\
\text { Function: } \\
\text { shoulder rating } \\
\text { questionnaire } \\
\text { Strength: } \\
\text { isometric strength } \\
\text { flexion, external } \\
\text { rotation, internal } \\
\text { rotation, } \\
\text { abduction using } \\
\text { dynamometer }\end{array}$ & Significant difference between groups \\
\hline
\end{tabular}


Table 3. Comparison of Exercise with other Modalities, Including Manual Therapy, Physiotherapy, Surgery and Injection

\begin{tabular}{|c|c|c|c|c|c|c|c|}
\hline Author & Participants & Age & Intervention & Frequency & Follow up & $\begin{array}{l}\text { Outcome } \\
\text { measures }\end{array}$ & Results \\
\hline $\begin{array}{l}\text { Cloke et al. } \\
\text { [9] }\end{array}$ & $\begin{array}{l}\mathrm{N}=112 \\
\text { (Male: } 48 \\
\text { Female : } 64)\end{array}$ & 22 to 88 & $\begin{array}{l}\text { 1. Manual therapy not } \\
\text { specified. } \\
\text { 2. Corticosteroid injection } \\
\text { 3. Combination of } 1 \text { and } 2\end{array}$ & $\begin{array}{c}6 \text { sessions over maximum } 18 \\
\text { weeks }\end{array}$ & $\begin{array}{l}18 \text { weeks } \\
\text { and } 1 \text { year }\end{array}$ & $\begin{array}{c}\text { Patient's } \\
\text { perceived } \\
\text { improvement: } \\
\text { rated better, same, } \\
\text { or worse and need } \\
\text { for surgery at } 1 \\
\text { year. } \\
\end{array}$ & $\begin{array}{l}\text { No major difference between groups at the conclusion of } \\
\text { the intervention or within } 1 \text { year of the follow-up }\end{array}$ \\
\hline $\begin{array}{l}\text { Conroy et } \\
\text { al. [10] }\end{array}$ & $\begin{array}{c}\mathrm{N}=14 \\
(\text { Male }: 8 \\
\text { Female }: 6)\end{array}$ & $\begin{array}{l}\text { Mean age } \\
52.9\end{array}$ & $\begin{array}{l}\text { 1. Manual therapy (n=7): } \\
\text { Joint mobilisations plus } \\
\text { comprehensive } \\
\text { physiotherapy } \\
\text { 2. Exercise therapy (n=7) } \\
\text { Active range of } \\
\text { motion-pendulum } \\
\text { exercises, cane-assisted } \\
\text { stretching into flexion and } \\
\text { external rotation, } \\
\text { towel-assisted internal } \\
\text { rotation, assisted horizontal } \\
\text { adduction Strengthening: } \\
\text { chair press, isometric } \\
\text { internal rotation, external } \\
\text { rotation, scapular } \\
\text { strengthening exercises } \\
\text { Stretching }\end{array}$ & $\begin{array}{l}3 \text { sessions ( } 45 \text { to } 60 \text { minutes)/ } \\
\text { week for } 3 \text { weeks }\end{array}$ & $\begin{array}{l}3 \text { weeks } \\
\text { post } \\
\text { treatment }\end{array}$ & $\begin{array}{l}\text { Pain: } 100 \mathrm{~mm} \\
\text { visual analogue } \\
\text { scale } \\
\text { Range of motion: } \\
\text { flexion, abduction, } \\
\text { Scaption, internal } \\
\text { rotation, external } \\
\text { rotation using } \\
\text { goniometer. } \\
\text { Overhead } \\
\text { function: graded } \\
\text { by examiner on } \\
\text { 3-point rating } \\
\text { scale }\end{array}$ & $\begin{array}{l}\text { Both groups have become better. The manual therapy } \\
\text { group dramatically improved with the subacromial } \\
\text { compression test on 24-hour pain and discomfort, but no } \\
\text { improvements in range of motion and function relative to } \\
\text { the exercise group. } \\
\text { On all factors, the Manual Therapy group improved, } \\
\text { while the exercise group only improved agility and } \\
\text { function. } \\
\text { 24-hour pain: Group 1vs Group 2: } 49.71 \text { (29.01) to } \\
\text { 12.5(14.93) vs } 48.07(21.33) \text { to } 45.86(33.26) \text { Pain with } \\
\text { subacromial compression test } \\
\text { Group 1vs Group 2: } 50.50 \text { (25.18) to } 22.43 \text { (14.13) vs } \\
56.71(31.42) \text { to } 53.57 \text { (32.86) }\end{array}$ \\
\hline $\begin{array}{l}\text { Dickens et } \\
\text { al. [13] }\end{array}$ & $\begin{array}{l}\mathrm{N}=85 \text { (Male: } \\
\text { 48; Female : } \\
\text { 37) }\end{array}$ & 27 to 68 & $\begin{array}{l}\text { 1. Individualised } \\
\text { rehabilitation programme } \\
\text { (n=45): based on findings } \\
\text { from initial assessment. } \\
\text { Modalities inclusive } \\
\text { mobilisation, postural } \\
\text { advice, strapping, } \\
\text { electrotherapy, progressive } \\
\text { exercise therapy (for } \\
\text { scapulothoracic and rotator } \\
\text { cuff muscles) with } \\
\text { Theraband } \\
\text { 2. No intervention }(\mathrm{n}=40)\end{array}$ & $\begin{array}{l}\text { 1: } 1 \text { or } 2 / \text { week and home } \\
\text { exercise programme }\end{array}$ & 6 months & $\begin{array}{l}\text { Patients opting for } \\
\text { surgery after } \\
\text { intervention } \\
\text { Constant Score }\end{array}$ & $\begin{array}{l}\text { Significantly lower opting surgery rates in the exercise } \\
\text { group ( } 74 \% \text { ) than in the control group (100\%) Mean } \\
\text { change in the exercise group is } 20 \text { ( } 4 \text { to } 45 \text { ) vs. } 0.65 \text { ( }-16 \\
\text { to -14) in the control group in the Constant Score group }\end{array}$ \\
\hline
\end{tabular}


Table 3 Continued

\begin{tabular}{|c|c|c|c|c|c|c|c|}
\hline $\begin{array}{c}\text { Engebretsen } \\
\text { et al. } \\
{[15,16]}\end{array}$ & $\begin{array}{c}\mathrm{N}=104 \\
\text { (Male:52; } \\
\text { Female :52) }\end{array}$ & 18 to 70 & $\begin{array}{l}\text { 1. Exercise group (same as } \\
\text { Brox et al. 1993, 1994) } \\
\text { 2. Radial ESWT group }\end{array}$ & $\begin{array}{l}\text { 1. Two } 45 \text { minute sessions } \\
\text { weekly plus home exercise } \\
\text { programme for up to } 12 \text { weeks } \\
\text { 2. 1/week x } 4 \text { to } 6 \text { weeks }\end{array}$ & $\begin{array}{c}6,12,18 \\
\text { week, } 1 \\
\text { year }\end{array}$ & $\begin{array}{l}\text { Pain: 9-point } \\
\text { rating scale } \\
\begin{array}{c}\text { Function: } 7 \text { point } \\
\text { rating scale }\end{array} \\
\text { Work status: } \\
\text { self-reported } \\
\text { questionnaire }\end{array}$ & $\begin{array}{l}\text { A beneficial effect was observed in favour of supervised } \\
\text { exercises at } 6,12 \text { and } 18 \text { weeks. The changed impact of } \\
\text { treatment was - } 84 \text { (-16.5 to -0.6). } \\
\text { Improved the slightly greater percentage of patients in } \\
\text { the population treated with supervised exercises, odd } \\
\text { ratio } 32 \text { ( } 13 \text { to } 7.8) \text {. In the ESWT group, more patients } \\
\text { received additional therapy between } 12 \text { and } 18 \\
\text { weeks-odd ratio of } 555 \text { (1.3 to } 26.4) \\
\text { At } 1 \text { year, there were no major variations between the } 2 \\
\text { primary outcome assessment classes (-7.6 points, } 95 \% \\
\text { confidence interval=-16.6 to } 0.5 \text { ) and the use of pain, } \\
\text { function and treatment. In contrast with the ESWT } \\
\text { category, the higher percentage in the exercise group } \\
\text { (60\%) was classified as clinically improved (52\% ) }\end{array}$ \\
\hline $\begin{array}{c}\text { Ginn et al. } \\
\text { [19] }\end{array}$ & $\begin{array}{c}\mathrm{N}=138 \\
\text { (Male: } 82 \text {; } \\
\text { Female: } 56 \text { ) }\end{array}$ & $>=18$ & $\begin{array}{l}\text { 1. Single subacromial } \\
\text { injection with } \\
\text { methylprednisone acetate } \\
\text { (n=48) } \\
\text { 2. Regular home-based } \\
\text { exercises for } 5 \text { weeks, } \\
\text { customised for each } \\
\text { patient, restoration } \\
\text { exercises } \\
\text { Dynamic flexibility (n=48) } \\
\text { and shoulder muscle } \\
\text { coordination, including } \\
\text { muscle stretching, poor } \\
\text { muscle strengthening, } \\
\text { motor re-training to } \\
\text { enhance scapula-humeral } \\
\text { rhythm, and muscle } \\
\text { coordination. } \\
\\
\text { 3. Electrophysical } \\
\text { modalities, passive joint } \\
\text { mobilisation, daily range of } \\
\text { motion exercises } \\
\text { (abduction, flexion, } \\
\text { extension, horizontal } \\
\text { flexion) against elastic } \\
\text { resistance (n=42) }\end{array}$ & $\begin{array}{c}\text { Group 2: } 5 / \text { weeks for } 5 \text { weeks; } \\
\text { supervised once a week for } \\
\text { progression Group 3: daily for } 5 \\
\text { weeks }\end{array}$ & 5 weeks & $\begin{array}{l}\text { Pain: } 100 \mathrm{~mm} \\
\text { visual analogue } \\
\text { scale function: } \\
\text { non-validated } \\
\text { questionnaire } \\
\text { Strength: } \\
\text { abduction strength } \\
\text { measured using } \\
\text { dynamometer } \\
\text { range of motion: } \\
\text { pain free flexion, } \\
\text { abduction } \\
\text { Self-assessed } \\
\text { improvement: } \\
\text { perceived change } \\
\text { in symptoms }\end{array}$ & $\begin{array}{l}\text { Both approaches were similarly successful in reducing } \\
\text { short-term pain. }\end{array}$ \\
\hline
\end{tabular}


Table 3 Continued

\begin{tabular}{|c|c|c|c|c|c|c|c|}
\hline $\begin{array}{l}\text { Kachingwe } \\
\text { et al. [25] }\end{array}$ & $\begin{array}{c}\mathrm{N}=33 \text { (Male: } \\
\text { 17; Female: } \\
\text { 16) }\end{array}$ & 18 to 74 & $\begin{array}{l}\text { 1. Supervised exercise: } \\
\text { posterior capsular } \\
\text { stretching, postural } \\
\text { correction exercises, rotator } \\
\text { cuff strengthening (elastic } \\
\text { resistance external } \\
\text { rotation), scapular stability } \\
\text { exercise (scapular exercises } \\
\text { with elastic resistance), ice } \\
\text { pack } \\
\text { 2. Supervised exercise as } \\
\text { per group } 1 \text { with } \\
\text { glenohumeral joint } \\
\text { mobilisations } \\
\text { 3. Supervised exercises as } \\
\text { per group } 1 \text { with technique } \\
\text { (Mulligan mobilisations). } \\
\text { 4. Control group with } \\
\text { advice only }\end{array}$ & $\begin{array}{c}\text { Group } 1 \text { to } 3 \text { received } \\
\text { physiotherapy } x 1 / \text { week x } 6 \\
\text { weeks with home exercise } \\
\text { programme daily }\end{array}$ & 6 weeks & $\begin{array}{l}\text { Pain: } 10 \text { point } \\
\text { visual analogue } \\
\text { scale. } \\
\text { Range of motion: } \\
\text { Goniometric } \\
\text { measurement of } \\
\text { flex and scaption } \\
\text { Function: shoulder } \\
\text { pain and disability } \\
\text { index }\end{array}$ & $\begin{array}{l}\text { Both classes got stronger. Manual therapy group } \\
\text { improved substantially on } 24 \text {-hour pain and discomfort } \\
\text { with subacromial compression test but no changes in } \\
\text { range of motion and function compared with exercise } \\
\text { group. The manual therapy group improved on all } \\
\text { variables while the exercise group improved on mobility } \\
\text { and work only. } \\
\text { Group } 2 \text { and } 3 \text { had higher } \% \text { change from pre-to post on } \\
\text { all } 3 \text { pain treatment measures than group } 1 \text { though not } \\
\text { statistically significant Group } 1 \text { vs Group 2: visual } \\
\text { analogue scale: } 20.8 \text { (112.3) \% vs } 44.2(38.6) \% \text { Neer: } \\
44.0 \text { (57.2) \% vs } 57.6 \text { (38.7) } \%: 39.5 \text { (54.9) } \% \text { vs } 52.1 \\
\text { (62.9) } \%\end{array}$ \\
\hline $\begin{array}{l}\text { Walther et } \\
\text { al. [46] }\end{array}$ & $\begin{array}{c}\mathrm{N}=60 \text { (Male: } \\
\quad 34 ; \\
\text { Female }: 26)\end{array}$ & 25 to 66 & $\begin{array}{l}\text { 1: Exercise: Standardised } \\
\text { self-training; centring and } \\
\text { stretching using elastic } \\
\text { resistance, scapular } \\
\text { stability with and without } \\
\text { elastic resistance. Weighted } \\
\text { pendulum exercises for } \\
\text { range of motion } \\
\text { 2: Conventional } \\
\text { physiotherapy: centring } \\
\text { training for the rotator cuff } \\
\text { and stretching with advice } \\
\text { 3: Functional brace }\end{array}$ & $\begin{array}{l}\text { Group 1: x4 sessions, plus } \\
\text { x5/weeks x } 10 \text { to } 15 \text { minutes } \\
\text { home exercise programme } \\
\text { Group 2: x } 10 \text { sessions-given } 2 \\
\text { to } 3 \text { sessions/week }\end{array}$ & $\begin{array}{c}6 \text { and } 12 \\
\text { weeks }\end{array}$ & $\begin{array}{l}\text { Pain: } 100 \mathrm{~mm} \\
\text { visual analogue } \\
\text { scale } \\
\text { Function: } \\
\text { Constant-Murley } \\
\text { scale }\end{array}$ & $\begin{array}{l}\text { All groups showed substantial pain and work } \\
\text { improvement No major differences between groups were } \\
\text { found }\end{array}$ \\
\hline $\begin{array}{c}\text { Haahr et al. } \\
\text { [21] }\end{array}$ & $\begin{array}{c}\mathrm{N}=90 \\
\text { (Male:32; } \\
\text { Female: } 58 \text { ) }\end{array}$ & 18 to 55 & $\begin{array}{l}\text { 1: Active training of } \\
\text { scapular stability, hot/ cold } \\
\text { packs and soft tissue } \\
\text { treatments } \\
\text { 2: Arthroscopic surgery }\end{array}$ & $\begin{array}{c}\text { Group 1: } 19 \text { sessions (x3/week } \\
\text { in first } 2 \text { weeks, } x 2 / \text { week for } \\
\text { next } 3 \text { weeks, x1/week in last } 7 \\
\text { weeks) }\end{array}$ & $\begin{array}{l}3,6,12 \\
\text { months }\end{array}$ & $\begin{array}{l}\text { Pain and function: } \\
\text { Constant-Murley } \\
\text { subscores 4-point } \\
\text { rating scale rating } \\
\text { pain and } \\
\text { dysfunction. } \\
\end{array}$ & No significant differences between groups \\
\hline
\end{tabular}


Table 3 Continued

\begin{tabular}{|c|c|c|c|c|c|c|c|}
\hline $\begin{array}{c}\text { Rahme et al. } \\
\text { [41] }\end{array}$ & $\begin{array}{l}\mathrm{N}=42 \\
\text { (Male: } 19 \\
\text { Female : } 23 \text { ) }\end{array}$ & 28 to 63 & $\begin{array}{l}\text { 1. Anterior acromioplasty + } \\
\text { post-op } \\
\text { physiotherapy (n=21): } \\
\text { 2.Physiotherapy (n=21) } \\
\text { 3. (n=12): from Group } 2 \\
\text { who opted for surgery } 6 \\
\text { months after physiotherapy } \\
\text { Exercises include unloaded } \\
\text { movement of shoulder, } \\
\text { measures to normalise the } \\
\text { scapulohumeral rhythm } \\
\text { and to increase postural } \\
\text { awareness, strengthening of } \\
\text { the shoulder muscles and } \\
\text { endurance training. } \\
\text { Submaximal training of } \\
\text { rotator cuff was started } \\
\text { about } 3 \text { months after the } \\
\text { operation in Group } 1 \text { and } 3\end{array}$ & $\mathrm{X} 2$ to 3 /weeks & $\begin{array}{l}6 \text { and } 12 \\
\text { months }\end{array}$ & $\begin{array}{l}\text { Visual analogue } \\
\text { scale } \\
\text { : reduction of } \\
\text { more than } 50 \% \\
\text { from initial score }\end{array}$ & $\begin{array}{c}6 \text { months: Group } 1 \text { had higher successful cases than } \\
\text { Group } 2(57 \% \text { vs } 33 \%) \text { but no } \\
\text { statistical analysis was done }\end{array}$ \\
\hline
\end{tabular}


Table 4. Comparison of Exercise with Exercise Combined with other Modalities Including Manual Therapy or Physiotherapy

\begin{tabular}{|c|c|c|c|c|c|c|c|}
\hline Author & Participants & Age & Intervention & Frequency & Follow up & Outcome measures & Results \\
\hline Bang et al. [2] & $\begin{array}{l}\mathrm{N}=52 \text { (Male: } 30 \\
\text { Female }: 22 \text { ) }\end{array}$ & 18 to 65 & $\begin{array}{l}\text { 1. Exercise }(\mathrm{n}=28) \\
\text { 2. Exercise+manual therapy }(\mathrm{n}=23) \\
\text { Supervised exercise: Flexibility: Anterior and } \\
\text { posterior shoulder stretches } \\
\text { Strengthening: seated press up, elbow push-up, } \\
\text { scapular exercises with elastic resistance }\end{array}$ & $\begin{array}{c}2 \times 30 \text { minutes/week } 3 \\
\text { weeks }\end{array}$ & $\begin{array}{c}\text { Post } \\
\text { treatment, } \\
2 \text { months } \\
\text { after } \\
\text { treatment }\end{array}$ & $\begin{array}{l}\text { Pain: } 100 \mathrm{~mm} \text { visual } \\
\text { analogue scale } \\
\text { Function: modified } \\
\text { Oswestry low back } \\
\text { disability } \\
\text { questionnaire }\end{array}$ & $\begin{array}{c}\text { Subjects in both groups } \\
\text { experienced significant } \\
\text { decreases in pain and } \\
\text { increases in function, but } \\
\text { there was significantly more } \\
\text { improvement in the manual } \\
\text { therapy group compared to } \\
\text { the exercise group. Pain in the } \\
\text { manual therapy group } \\
\text { reduced from a pre-treatment } \\
\text { mean of } 575.8 \text { (272.3) to a } \\
\text { post treatment mean of } \\
\text { 174.4(183.1), In contrast, } \\
\text { pain in the exercise group was } \\
\text { reduced from a pre-treatment } \\
\text { mean of 557.1 (237.2) to post } \\
\text { treatment mean of } 360.6 \\
\text { (272.3). Strength in the } \\
\text { manual therapy group } \\
\text { improved significantly while } \\
\text { strength in the exercise group } \\
\text { did not. }\end{array}$ \\
\hline Bennell et al. [3] & $\begin{array}{l}\text { N=120 (Male: } \\
\text { 74; Female: 46) }\end{array}$ & $>=18$ & $\begin{array}{l}\text { 1. Control group: placebo-inactive ultrasound } \\
\text { therapy+ } 10 \text { sessions of individual } \\
\text { standardised treatment over } 10 \text { weeks } \\
\text { 2. Active treatment group: } 10 \text { sessions of } \\
\text { individual standardised treatment over } 10 \\
\text { weeks } \\
\text { manual therapy and home exercises } \\
\text { Exercises: A progressive programme of } \\
\text { scapular stability retraining, rotator cuff and } \\
\text { scapular muscle resisted exercises against } \\
\text { elastic resistance or hand weights, and } \\
\text { shoulder girdle and thoracic spine flexibility } \\
\text { exercises. All resistance exercises progressed } \\
\text { by increasing repetitions, resistance, and } \\
\text { working rotator cuff muscles through range to } \\
90^{\circ} \text { abduction }\end{array}$ & $\begin{array}{l}10 \text { sessions for } 10 \text { weeks } \\
\text { Group } 2 \text { performed home } \\
\text { exercises for } 12 \text { weeks }\end{array}$ & $\begin{array}{l}11 \text { and } 22 \\
\text { weeks }\end{array}$ & $\begin{array}{l}\text { Pain: numeric pain } \\
\text { rating scale and visual } \\
\text { analogue scale }\end{array}$ & $\begin{array}{l}11 \text { weeks: Both groups } \\
\text { significantly improved with } \\
\text { decreased pain and increased } \\
\text { function but no difference } \\
\text { was found between groups } \\
22 \text { weeks: Active treatment } \\
\text { group showed a significantly } \\
\text { greater improvement in } \\
\text { shoulder pain and disability } \\
\text { index than the control group } \\
\text { (between group difference } \\
7.1,0.3 \text { to } 13.9 \text { ) but no } \\
\text { significant difference existed } \\
\text { between groups for change in } \\
\text { pain }(0.9,-0.03 \text { to } 1.7) \text { or for } \\
\text { percentage of participants } \\
\text { reporting a successful } \\
\text { treatment outcome (42 \% vs } \\
30 \% \text { ) }\end{array}$ \\
\hline
\end{tabular}


Table 4 Continued

\begin{tabular}{|c|c|c|c|c|c|c|c|}
\hline Senbursa et al. [44] & $\mathrm{N}=30$ & 30 to 55 & $\begin{array}{l}\text { 1. Home exercise }(\mathrm{n}=15) \text { home exercise } \\
\text { programme }(10 \text { to } 15 \text { minutes): Stretching and } \\
\text { strength exercises of rotator cuff, rhomboids, } \\
\text { levator scapulae, and serratus anterior using } \\
\text { elastic band } \\
\text { 2. Combined physiotherapy group manual } \\
\text { therapy (joint and soft tissue mobilisations, and } \\
\text { proprioceptive neuromuscular } \\
\text { facilitation)+home exercise }(\mathrm{n}=15)\end{array}$ & $\begin{array}{l}\text { Group 1: home exercise } \\
\text { programme for } 4 \text { weeks } \\
\text { Group 2: x3/weeks for } 4 \\
\text { weeks }\end{array}$ & $\begin{array}{l}\text { Post } \\
\text { treatment } \\
\text { and } 3 \\
\text { months } \\
\text { after } \\
\text { initiation of } \\
\text { treatment }\end{array}$ & $\begin{array}{l}\text { Pain: } 10 \mathrm{~cm} \text { visual } \\
\text { analogue scale active } \\
\text { range of motion: } \\
\text { goniometry flexion, } \\
\text { abduction, internal } \\
\text { rotation, external } \\
\text { rotation Function: } \\
\text { Neer Questionnaire }\end{array}$ & $\begin{array}{l}\text { Subjects in both groups had } \\
\text { significant decrease in pain } \\
\text { and increase in shoulder } \\
\text { function. But there was } \\
\text { significantly more } \\
\text { improvement in the manual } \\
\text { therapy group compared to } \\
\text { the exercise group. Change in } \\
\text { pain in manual therapy vs } \\
\text { exercise group: } 6.7(0.3) \text { to } \\
2.0(2.0) \text { vs } 6.6(1.4) \text { to } 3.0 \\
(1.8) \text { range of motion at } \\
\text { flexion, abduction and } \\
\text { external rotation in the } \\
\text { manual therapy group } \\
\text { improved significantly while } \\
\text { Range of motion in the } \\
\text { exercise group did not. } \\
\text { Combined physiotherapy } \\
\text { group showed significantly } \\
\text { greater improvement in the } \\
\text { Neer Questionnaire score and } \\
\text { shoulder satisfaction score } \\
\text { than home exercise group. }\end{array}$ \\
\hline $\begin{array}{c}\text { Yiasemides et } 1 . \\
{[48]}\end{array}$ & $\mathrm{N}=98$ & 27 to 85 & $\begin{array}{l}\text { 1. Control exercise group }(\mathrm{n}=51) \text { : Advice and } \\
\text { exercises including stretching, strengthening } \\
\text { of weakened muscles and restoring } \\
\text { scapulahumeral rhythm, motor retraining } \\
\text { 2. Experimental group }(\mathrm{n}=47) \text { : Low velocity } \\
\text { passive joint mobilisation to glenohumeral } \\
\text { joint, sternoclavicular joint, acromioclavicular } \\
\text { joint }\end{array}$ & $\begin{array}{l}\text { Daily home based } \\
\text { programme for } 12 \text { weeks } \\
\text { and reviewed by } \\
\text { physiotherapist } 1 \text { to } \\
\text { 2/weeks }\end{array}$ & $\begin{array}{l}1,3,6 \\
\text { months }\end{array}$ & $\begin{array}{l}\text { Shoulder pain and } \\
\text { disability index } \\
\text { Self-rate change active } \\
\text { range of motion }\end{array}$ & $\begin{array}{l}\text { No statistically significant } \\
\text { differences were detected in } \\
\text { any outcome measures in the } \\
\text { follow up }\end{array}$ \\
\hline
\end{tabular}


Table 5. Comparison of ESWT with Sham ESWT

\begin{tabular}{|c|c|c|c|c|c|c|c|}
\hline Author & Participants & Age & Intervention & Frequency & Follow up & Outcome measures & Results \\
\hline Galasso et al. [17] & $\begin{array}{c}\mathrm{N}=20 \\
\text { Noncalcifying } \\
\text { supraspinatus } \\
\text { tendinopathy }\end{array}$ & $\begin{array}{c}\text { Mean } \\
\text { age } \\
50\end{array}$ & $\begin{array}{c}\text { Group } 1(\mathrm{n}=11) \text { : } \\
\text { ESWT 2x3000 } \\
\text { Group } 2(\mathrm{n}=9) \text { : Sham } \\
\text { ESWT }\end{array}$ & $\begin{array}{c}\text { 1-week interval/ } \\
\text { energy flux density: } \\
0.068 \mathrm{~mJ} / \mathrm{mm} 2\end{array}$ & $\begin{array}{c}6,12 \text { week, } \\
9 \text { years }\end{array}$ & $\begin{array}{l}\text { Constant and Murley } \\
\text { score } \\
\text { range of motion } \\
\text { The definition of } \\
\text { success rate } \\
\text { same as Schmitt } \\
(2001)\end{array}$ & $\begin{array}{c}\text { Significantly higher Constant and } \\
\text { Murley score } \\
\text { and range of motion in Group } 1 \\
\text { when compared with Group } 2 \\
\text { Constant score } \\
\text { Control vs treatment group: } \\
\text { Pre-treatment: } 42.45(9.83) \text { vs } \\
41.67(12.53) \\
\text { At } 6 \text { weeks: } 43.11(19.16) \text { vs } 64 . \\
(16.6) \\
\text { At } 12 \text { weeks: } 48(22.3) \text { vs } 74.09 \\
(20.56) \\
\text { Success rate } 3 \text { months post } \\
\text { treatment } \\
\text { Success rate: } 63.7 \% \text { vs } 22.3 \% \\
\\
\text { A telephone recall of ESWT } \\
\text { patients has been carried out nine } \\
\text { years after treatment and were able } \\
\text { to reach } 10 \text { out of } \\
11 \text { individuals, all patients were } \\
\text { satisfied with the } \\
\text { treatment received and would have } \\
\text { repeated the same } \\
\text { therapy again. }\end{array}$ \\
\hline Schmitt et al. [43] & $\begin{array}{c}\mathrm{N}=40 \\
\text { Noncalcific } \\
\text { supraspinatus } \\
\text { tendinosis }\end{array}$ & $\begin{array}{c}\text { Mean age } \\
52 \\
\text { years } \\
(29 \text { to } \\
66)\end{array}$ & $\begin{array}{c}\text { Group } 1(\mathrm{n}=20) \text { : } \\
\text { ESWT } \\
3 \times 2000 \\
\text { Group 2 }(\mathrm{n}=20) \text { : Sham } \\
\text { ESWT }\end{array}$ & $\begin{array}{c}\text { Group 1: } 1 \text { week } \\
\text { interval/ } \\
0.11 \mathrm{~mJ} / \mathrm{mm} 2\end{array}$ & 6.12 week & $\begin{array}{c}\text { Pain: 11-point } \\
\text { constant score } \\
\text { patient were } \\
\text { considered a } \\
\text { treatment success if } \\
\text { they } \\
\text { showed an } \\
\text { improvement of at } \\
\text { least } 30 \text { points, or } \\
\text { their Constant } \\
\text { Murley score at the } \\
\text { endpoint of the study } \\
\text { was at least } 80 \% \text { of } \\
\text { the standard age and } \\
\text { gender-related value. }\end{array}$ & $\begin{array}{c}\text { No significant difference between } \\
\text { groups was found. } \\
\text { Constant score } \\
\text { Control vs treatment group: } \\
\text { Pre-treatment: } 42.20(13.04) \text { vs } \\
40.70(13.29) \\
\text { At } 6 \text { weeks: } 64.17(25.17) \text { vs } \\
60.95(29.62) \\
\text { At } 12 \text { weeks: } 64.39(32.68) \text { vs } \\
66.50(37.92) \\
\text { Success rate: } 44 \% \text { vs } 50 \%\end{array}$ \\
\hline
\end{tabular}


Table 6. Effects of ESWT on pain and Oxygen saturation

\begin{tabular}{|c|c|c|c|c|c|c|c|}
\hline Author & Participants & Age & Intervention & Frequency & Follow up & $\begin{array}{l}\text { Outcome } \\
\text { measures }\end{array}$ & Results \\
\hline Notarincola et al. [38] & $\mathrm{N}=30$ & 45 to 78 & & $\begin{array}{c}3 \times 2000,3 \text { to } 4 \text { days } \\
\text { interval/ } \\
0.04 \text { to } 0.07 \\
\mathrm{~mJ} / \mathrm{mm} 2\end{array}$ & $\begin{array}{c}\text { 1st, 2nd, 3rd } \\
\text { session of } \\
\text { ESWT, } 2 \\
\text { months, } \\
6 \text { months }\end{array}$ & $\begin{array}{c}\text { Pain: visual } \\
\text { analogue scale } \\
\text { constant Score }\end{array}$ & $\begin{array}{c}\text { A clinical improvement was obtained } \\
\text { in } 65.6 \% \text { of patients } \\
\text { at } 2 \text { and } 6 \text { months. This was associated } \\
\text { with statistically } \\
\text { significant reduction in the oxygen } \\
\text { tissue saturation, } \\
\text { during treatment as well as at } \\
\text { subsequent follow-up visits }\end{array}$ \\
\hline
\end{tabular}

Table 7. Comparison of ESWT plus Kinesitherapy vs Kinesitherapy vs Postural Hygiene

\begin{tabular}{|c|c|c|c|c|c|c|c|}
\hline Author & Participants & Age & Intervention & Frequency & $\begin{array}{c}\text { Follow } \\
\text { up }\end{array}$ & $\begin{array}{l}\text { Outcome } \\
\text { measures }\end{array}$ & Results \\
\hline Melegati et al. [34] & $\begin{array}{l}\quad \mathrm{N}=90 \\
\text { Noncalcific } \\
\text { subacromia } \\
\text { impingement } \\
\text { syndrome }\end{array}$ & $\begin{array}{c}\text { Not } \\
\text { reported }\end{array}$ & $\begin{array}{c}\text { Group } 1(\mathrm{n}=30) \text { : } \\
\text { Kinesitherapy sessions } \\
\text { (Exercise: Codman, } \\
\text { capsular stretching, } \\
\text { isometric for rotators and } \\
\text { deltoid, elastic resistance } \\
\text { for rotator cuff, deltoid and } \\
\text { trapezius) } \\
\text { Group } 2 \text { (n=30): medium } \\
\text { ESWT 3x2000 pulses } \\
\text { followed by } \\
\text { kinesitherapy session } \\
\text { Group } 3 \text { (n=30): Control, } \\
\text { one session postural } \\
\text { hygiene and joint } \\
\text { economy suggestion }\end{array}$ & $\begin{array}{c}\text { Group 1: } 6 \mathrm{x} \text { at } 3 \\
\text { week interval } \\
\text { Group 2: } 1 \text { week } \\
\text { interval/ } 0.22 \\
\mathrm{~mJ} / \mathrm{mm} 2\end{array}$ & 80 days & $\begin{array}{c}\text { Improvement } \\
\text { in constant } \\
\text { score }\end{array}$ & $\begin{array}{l}\text { Significant improvement within } \\
\text { Group } 1(\mathrm{p}<0.0001) \text { and } \\
\text { Group 2 } \mathrm{p}<0.0001) \text {. } \\
\text { Significant better Constant score in } \\
\text { Group 1 and Group 2 } \\
\text { when compared with Group 3: } \\
\text { Group } 2 \text { vs Group 1: } 27.95 \% \text { (in } \\
\text { favour of Group 2) } \\
\text { Group } 2 \text { vs Group 3: 80.41\% (in } \\
\text { favour of Group 2) } \\
\text { Group } 1 \text { vs Group 3: 72.81\% (in } \\
\text { favour of Group 1) }\end{array}$ \\
\hline
\end{tabular}


Table 8. Using Bodyblade Exercise

\begin{tabular}{|c|c|c|c|c|c|c|c|}
\hline Author & Participants & Age & Intervention & Frequency & Follow up & Outcome measures & Results \\
\hline Buteau et al. [8] & $\begin{array}{c}\mathrm{N}=1 \\
\text { male } \\
\text { suffered } \\
\text { from } \\
\text { glenohumeral } \\
\text { dislocation }\end{array}$ & 18 & $\begin{array}{l}\text { Bodyblade exercise } \\
\text { session: } 5 \text { minutes of warm up } \\
\text { on arm ergometer, each } \\
\text { session consists of } 4 \text { to } 7 \\
\text { movements with } 5 \text { to } 10 \text { reps x } \\
1 \text { to } 2 \text { sets; the exercises } \\
\text { progressed from scapula } \\
\text { control, unilateral upper } \\
\text { extremity in pure anatomical } \\
\text { movement, diagonal pattern; } \\
\text { feet off ground with fit ball } \\
\text { plus torso flexion, extension }\end{array}$ & $\begin{array}{c}12 \text { sessions } \\
\text { over } 4 \text { to } 6 \\
\text { weeks } \\
\text { sessions } 4 \text { to } 6\end{array}$ & $\begin{array}{l}\text { 6th, post } \\
\text { treatment, } 6 \text { months } \\
\text { post treatment }\end{array}$ & $\begin{array}{l}\text { Pain: 11-point } \\
\text { numeric pain active } \\
\text { range of motion } \\
\text { shoulder strength } \\
\text { with handheld } \\
\text { dynamometer } \\
\text { Pain and function: } \\
\text { shoulder pain and } \\
\text { disability index and } \\
\text { Western Ontario } \\
\text { shoulder index }\end{array}$ & $\begin{array}{c}\text { Pain decreased from } 4 \text { to } 0 \text { at } \\
\text { discharge } \\
\text { Range of motion and strength } \\
\text { improved } \\
\text { shoulder pain and disability } \\
\text { index } \\
\text { Decrease of } 11 \text { points which } \\
\text { indicates a meaningful clinical } \\
\text { change Western Ontario } \\
\text { shoulder index decreased from } \\
129 \text { to } 46 \\
\\
6 \text { months: no recurrent } \\
\text { dislocation was } \\
\text { reported }\end{array}$ \\
\hline Lee \& Yoo [29] & $\begin{array}{c}\mathrm{N}=18, \text { ice } \\
\text { hockey } \\
\text { players }\end{array}$ & $\begin{array}{c}\text { Group1 } \\
: \text { mean } \\
25.56 \pm \\
1.33 \\
\text { Group } \\
2: \text { mean } \\
26.56 \pm \\
2.83\end{array}$ & $\begin{array}{c}\text { Group 1: Players without } \\
\text { shoulder instability (control) } \\
\text { (n=9) } \\
\text { Group 2: Players with } \\
\text { shoulder instability ( } \mathrm{n}=9 \text { ) } \\
\text { training programme using } \\
\text { elastic bands (five exercises: } \\
\text { horizontal abduction, adduction, } \\
\text { internal rotation, external rotation } \\
\text { scapular retraction and } \\
\text { protraction at } 12 \text { repetition } \\
\text { maximum } 10 \text { to } 20 \\
\text { for } 40 \text { to } 50 \text { minutes) and } \\
\text { Bodyblade (4 exercises: } \\
\text { horizontal abduction, front } \\
\text { horizontal, scapular } \\
\text { retraction and stability } \\
\text { trainer push-up; } 5 \text { sets } \mathrm{x} \\
1 \text { minute) }\end{array}$ & $\begin{array}{l}4 \mathrm{x} / \text { week } \\
8 \text { weeks }\end{array}$ & Week $0,4,8$ & $\begin{array}{l}\text { Pain: } 100 \mathrm{~mm} \text { visual } \\
\text { analogue scale } \\
\text { isokinetic strength } \\
\text { on flexion, } \\
\text { extension, internal } \\
\text { rotation, external } \\
\text { rotation }\end{array}$ & $\begin{array}{c}\text { There were interactions in } \\
\text { flexion, extension, internal } \\
\text { rotation } \\
\text { and subjective pain score }\end{array}$ \\
\hline
\end{tabular}




\section{Discussion}

For non-operational care, there are a variety of therapies available, ranging from physical exercises to electrotherapy modalities that help to relieve pain and improve function. Many of the workout programmes are calming, reinforcing exercises aimed at normalising the operation of the scapular and glenohumeral $[11,53]$. The beneficial impact of exercise on pain and post-treatment function has previously been shown [1,22].

In order to improve pain reduction $[32,40]$ and work $[16,40,32.48]$ from week 4 through week 24 , a daily workout routine comprising of soft tissue calming and positive affirmation exercises has been published [49]. Centred on Holmgrens et al. [24], high-dose exercises involving federal, semi-national, national and aerobic shoulder exercises have demonstrated greater functional progress than the low-dose exercise category [40]. In physical events such as tossing at baseball and tennis, the energy is thought to be passed from the lower limbs from the trunk to the elbow. The operation of the shoulder will be affected by some weakening of the lower extremities or trunk muscles [45]. Exercise treatment involving stretching, glenohuermal and relaxation of the scapular muscles has been found to be effective for pain management and functional recovery 1 year after intervention [40,52]. The result is equivalent to that of 1 year post-intervention corticosteroid injection [9] and arthoscopic surgery $[6,21]$. The exercise category, which was tested after two years, demonstrated greater functional progress compared to placebo [6], which was close to the results for arthoscopic surgery $[26,27]$.

The functional improvements observed ranged from 50.8 to 81 percent $[15,21]$, despite these promising results. A stretching method for posterior capsules has reported an improvement in subacormial space in a group of overhead athletes with 27 glenohumeral inner rotation deficits [33]. However, Savoie et al. [42] and Desmeules et al. [12] found only the subacormic space to be increased in those patients with decreased subacormic space prior to recovery. As a result, a therapeutic or ergonomic exercise involving people with limited physical space may be more likely to be successful for this purpose. In patients who tend to be sedentary, the effectiveness of traditional therapy has shown its effects. The Bodyblade is a fairly new approach to rehabilitating shoulder-dysfunction athletes by strengthening and stretching their soft tissues. With repetitive forceful arm and shoulder contractions, the bar tips are able to move around. The muscles of the human body have to contract rapidly and therefore have to undergo up to 270 contractions per minute. It is known that externally induced vibration and oscillatory devices on the skeletal muscles have been documented to be used to enhance neuromuscular efficiency and induce short and long-term neurogenic adaptations [4,5]. Increased activation of primary movers, improved inter- and intramuscular synchronisation, enhanced synergistic synchronisation, and improved proprioception responses have been the potential benefits of vibration therapy. A study by Lister et al. [30] found that when a body weight equated as 35 pounds was placed on the upper chest and shoulder blades, the activation of the upper trapezius plus lower trapezius and anterior serratus was greater than the square of the body weight when the shoulder was flexed and abducted.

The effectiveness of middle-age localised ESWT on SAPS is not altogether proven. The effectiveness of its influence has not been tested on overhead athletes, who are younger in age and have a greater demand for tendons. Furthermore, all the studies used symptoms and subjective satisfaction as their main results, apart from the study by Notarnicola et al. [38]. Wang et al. [47] have also seen the neoangiogenic impact on the Achilles tendon caused by ESWT. For patients with subacromial tendinopathy, the therapeutic mechanism may be based on ESWT-mediated vascularisation, with the most recent results from Notarnicola et al. [38].

\section{Conclusions}

On the basis of these few experiments, exercise was found to be better than electrophysical modalities such as radial ESWT. It was also noted that the outcome of exercise therapy was similar to surgery. Exercise combined with manual therapy found that functional change, as well as an operation technique that had the same effects, was better than exercise alone. The efficacy of ESWT for shoulder SAPS has been unclear. To substantiate the effectiveness of Bodyblade exercise with SAPS, a wider range of cohorts with their performance quality compared to the conventional physiotherapy method will be essential. This knowledge enables better tracking of SAPS overhead athletes at the start of the conservation intervention.

\section{Competing Interests}

The authors declare that they have no competing interests.

\section{REFERENCES}

[1] Abdulla SY, Southerst D, Côté P, Shearer HM, Sutton D, Randhawa K, Varatharajan S, Wong JJ, Yu H, Marchand AA, Chrobak K. Is exercise effective for the management of subacromial impingement syndrome and other soft tissue injuries of the shoulder? A systematic review by the Ontario Protocol for Traffic Injury Management (OPTIMa) Collaboration. Manual therapy. 2015 Oct 1;20(5):646-56.

[2] Bang MD, Deyle GD. Comparison of supervised exercise 
with and without manual physical therapy for patients with shoulder impingement syndrome. Journal of Orthopaedic \& Sports Physical Therapy. 2000 Mar;30(3):126-37.

[3] Bennell K, Wee E, Coburn S, Green S, Harris A, Staples M, Forbes A, Buchbinder R. Efficacy of standardised manual therapy and home exercise programme for chronic rotator cuff disease: randomised placebo controlled trial. Bmj. 2010 Jun $8 ; 340$.

[4] Bosco C, Colli R, Introini E, Cardinale M, Tsarpela O, Madella A, Tihanyi J, Viru A. Adaptive respsonses of human skeletal muscle to vibration exposure. Clinical physiology. 1999 Mar 1;19(2):183.

[5] Bosco C, Iacovelli M, Tsarpela O, Cardinale M, Bonifazi M, Tihanyi J, Viru M, De Lorenzo A, Viru A. Hormonal responses to whole-body vibration in men. European journal of applied physiology. 2000 Mar 1;81(6):449-54.

[6] Brox JI, Gjengedal E, Uppheim G, Bøhmer AS, Brevik JI, Ljunggren AE, Staff PH. Arthroscopic surgery versus supervised exercises in patients with rotator cuff disease (stage II impingement syndrome): a prospective, randomized, controlled study in 125 patients with a 212 -year follow-up. Journal of shoulder and elbow surgery. 1999 Mar $1 ; 8(2): 102-11$.

[7] Brox JI, Staff PH, Ljunggren AE, Brevik JI. Arthroscopic surgery compared with supervised exercises in patients with rotator cuff disease (stage II impingement syndrome). British Medical Journal. 1993 Oct 9;307(6909):899-903.

[8] Buteau JL, Eriksrud O, Hasson SM. Rehabilitation of a glenohumeral instability utilizing the body blade. Physiotherapy theory and practice. 2007 Jan 1;23(6):333-49.

[9] Cloke DJ, Watson H, Purdy S, Steen IN, Williams JR. A pilot randomized, controlled trial of treatment for painful arc of the shoulder. Journal of shoulder and elbow surgery. 2008 Jan 1;17(1):S17-21.

[10] Conroy DE, Hayes KW. The effect of joint mobilization as a component of comprehensive treatment for primary shoulder impingement syndrome. Journal of Orthopaedic \& Sports Physical Therapy. 1998 Jul;28(1):3-14.

[11] Cools AM, Witvrouw EE, Declercq GA, Danneels LA, Cambier DC. Scapular muscle recruitment patterns: trapezius muscle latency with and without impingement symptoms. The American journal of sports medicine. 2003 Jul;31(4):542-9.

[12] Desmeules F, Minville L, Riederer B, Côté CH, Frémont P. Acromio-humeral distance variation measured by ultrasonography and its association with the outcome of rehabilitation for shoulder impingement syndrome. Clinical journal of sport medicine. 2004 Jul 1;14(4):197-205.

[13] Dickens SH, Cho BH. Interpretation of bond failure through conversion and residual solvent measurements and Weibull analyses of flexural and microtensile bond strengths of bonding agents. Dental Materials. 2005 Apr 1;21(4):354-64.

[14] Eckenrode BJ, Kelley MJ. Clinical biomechanics of the shoulder complex. InThe Athlete's Shoulder 2009 Jan 1 (pp. 17-41). Churchill Livingstone.

[15] Engebretsen K, Grotle M, Bautz-Holter E, Ekeberg OM, Juel NG, Brox JI. Supervised exercises compared with radial extracorporeal shock-wave therapy for subacromial shoulder pain: 1-year results of a single-blind randomized controlled trial. Physical therapy. 2011 Jan 1;91(1):37-47.

[16] Engebretsen K, Grotle M, Bautz-Holter E, Sandvik L, Juel NG, Ekeberg OM, Brox JI. Radial extracorporeal shockwave treatment compared with supervised exercises in patients with subacromial pain syndrome: single blind randomised study. Bmj. 2009 Sep 15;339:b3360.

[17] Galasso O, Amelio E, Riccelli DA, Gasparini G. Short-term outcomes of extracorporeal shock wave therapy for the treatment of chronic non-calcific tendinopathy of the supraspinatus: a double-blind, randomized, placebo-controlled trial. BMC musculoskeletal disorders. 2012 Dec 1;13(1):86

[18] Gerdesmeyer L, Wagenpfeil S, Haake M, Maier M, Loew M, Wörtler K, Lampe R, Seil R, Handle G, Gassel S, Rompe JD. Extracorporeal shock wave therapy for the treatment of chronic calcifying tendonitis of the rotator cuff: a randomized controlled trial. Jama. 2003 Nov 19;290(19):2573-80.

[19] Ginn K, Cohen M. Exercise therapy for shoulder pain aimed at restoring neuromuscular control: a randomized comparative clinical trial. Journal of Rehabilitation Medicine. 2005 Mar 1;37(2):115-22.

[20] Granviken F, Vasseljen O. Home exercises and supervised exercises are similarly effective for people with subacromial impingement: a randomised trial. Journal of physiotherapy. 2015 Jul 1;61(3):135-41.

[21] Haahr JP, Østergaard S, Dalsgaard J, Norup K, Frost P, Lausen S, Holm EA, Andersen JH. Exercises versus arthroscopic decompression in patients with subacromial impingement: a randomised, controlled study in 90 cases with a one year follow up. Annals of the rheumatic diseases. 2005 May 1;64(5):760-4.

[22] Hanratty CE, McVeigh JG, Kerr DP, Basford JR, Finch MB, Pendleton A, Sim J. The effectiveness of physiotherapy exercises in subacromial impingement syndrome: a systematic review and meta-analysis. InSeminars in arthritis and rheumatism 2012 Dec 1 (Vol. 42, No. 3, pp. 297-316). WB Saunders.

[23] Harryman 2nd DT, Sidles JA, Clark JM, McQuade KJ, Gibb TD, Matsen 3rd FA. Translation of the humeral head on the glenoid with passive glenohumeral motion. JBJS. 1990 Oct 1;72(9):1334-43.

[24] Holmgren T, Hallgren HB, Öberg B, Adolfsson L, Johansson K. Effect of specific exercise strategy on need for surgery in patients with subacromial impingement syndrome: randomised controlled study. Bmj. 2012 Feb 20;344:e787.

[25] Kachingwe AF, Phillips B, Sletten E, Plunkett SW. Comparison of manual therapy techniques with therapeutic exercise in the treatment of shoulder impingement: a randomized controlled pilot clinical trial. Journal of manual \& manipulative therapy. 2008 Oct 1;16(4):238-47.

[26] Ketola S, Lehtinen J, Arnala I, Nissinen M, Westenius H, Sintonen H, Aronen P, Konttinen YT, Malmivaara A, Rousi T. Does arthroscopic acromioplasty provide any additional value in the treatment of shoulder impingement syndrome? A two-year randomised controlled trial. The Journal of bone and joint surgery. British volume. 2009 Oct;91(10):1326-34. 
[27] Ketola S, Lehtinen J, Rousi T, Nissinen M, Huhtala H, Konttinen YT, Arnala I. No evidence of long-term benefits of arthroscopic acromioplasty in the treatment of shoulder impingement syndrome: five-year results of a randomised controlled trial. Bone \& joint research. 2013 Jul;2(7):132-9.

[28] Kibler WB, Sciascia A. Rehabilitation of the athlete's shoulder. Clinics in sports medicine. 2008 Oct $1 ; 27(4): 821-31$

[29] Lee C, Yoo J. The effects of a rehabilitation program for ice hockey players with shoulder injury. Journal of Physical Therapy Science. 2013;25(2):177-81.

[30] Lister JL, Del Rossi G, Ma F, Stoutenberg M, Adams JB, Tobkin S, Signorile JF. Scapular stabilizer activity during Bodyblade ${ }^{\circledR}$, cuff weights, and Thera-Band $\AA$ use. Journal of sport rehabilitation. 2007 Feb 1;16(1):50-67.

[31] Lombardi Jr I, Magri AG, Fleury AM, Da Silva AC, Natour J. Progressive resistance training in patients with shoulder impingement syndrome: a randomized controlled trial. Arthritis Care \& Research: Official Journal of the American College of Rheumatology. 2008 May 15;59(5):615-22.

[32] Ludewig PM, Borstad JD. Effects of a home exercise programme on shoulder pain and functional status in construction workers. Occupational and environmental medicine. 2003 Nov 1;60(11):841-9.

[33] Maenhout A, Van Eessel V, Van Dyck L, Vanraes A, Cools A. Quantifying acromiohumeral distance in overhead athletes with glenohumeral internal rotation loss and the influence of a stretching program. The American journal of sports medicine. 2012 Sep;40(9):2105-12.

[34] Melegati G, Tornese D, Bandi M. Effectiveness of extracorporeal shock wave therapy associated with kinesitherapy in the treatment of subacromial impingement: a randomised, controlled study. Journal of Sports Traumatology and Related Research. 2000 Jun 1;22(2):58-64.

[35] Myers JB, Hwang JH, Pasquale MR, Blackburn JT, Lephart SM. Rotator cuff coactivation ratios in participants with subacromial impingement syndrome. Journal of science and medicine in sport. 2009 Nov 1;12(6):603-8.

[36] Myers JB, Laudner KG, Pasquale MR, Bradley JP, Lephart SM. Glenohumeral range of motion deficits and posterior shoulder tightness in throwers with pathologic internal impingement. The American journal of sports medicine. 2006 Mar;34(3):385-91.

[37] Neer CS. Impingement lesions. Clinical Orthopaedics and Related Research (1976-2007). 1983 Mar 1;173:70-7.

[38] Notarnicola A, Moretti L, Tafuri S, Forcignanò M, Pesce V, Moretti B. Reduced local perfusion after shock wave treatment of rotator cuff tendinopathy. Ultrasound in medicine \& biology. 2011 Mar 1;37(3):417-25.

[39] Ogden JA, Alvarez RG, Levitt R, Marlow M. Shock wave therapy (Orthotripsy®) in musculoskeletal disorders. Clinical Orthopaedics and Related Research®. 2001 Jun $1 ; 387: 22-40$.

[40] Østerås H, Torstensen TA, Haugerud L, Østerås BS. Doseresponse effects of graded therapeutic exercises in patients with long-standing subacromial pain. Advances in physiotherapy. 2009 Jan 1;11(4):199-209.

[41] Rahme H, Solem-Bertoft E, Westerberg CE, Lundberg E, Sörensen S, Hilding S. The subacromial impingement syndrome. A study of results of treatment with special emphasis on predictive factors and pain-generating mechanisms. Scandinavian journal of rehabilitation medicine. 1998 Dec 1;30(4):253-62.

[42] Savoie A, Mercier C, Desmeules F, Frémont P, Roy JS Effects of a movement training oriented rehabilitation program on symptoms, functional limitations and acromiohumeral distance in individuals with subacromial pain syndrome. Manual therapy. 2015 Oct 1;20(5):703-8.

[43] Schmitt J, Haake M, Tosch A, Hildebrand R, Deike B, Griss P. Low-energy extracorporeal shock-wave treatment (ESWT) for tendinitis of the supraspinatus: a prospective, randomised study. The Journal of Bone and Joint Surgery. British volume. 2001 Aug;83(6):873-6.

[44] Senbursa G, Baltacı G, Atay A. Comparison of conservative treatment with and without manual physical therapy for patients with shoulder impingement syndrome: a prospective, randomized clinical trial. Knee surgery, sports traumatology, arthroscopy. $2007 \mathrm{Jul}$ 1;15(7):915-21.

[45] Seroyer ST, Nho SJ, Bach BR, Bush-Joseph CA, Nicholson GP, Romeo AA. The kinetic chain in overhand pitching: its potential role for performance enhancement and injury prevention. Sports health. 2010 Mar;2(2):135-46.

[46] Walther M, Werner A, Stahlschmidt T, Woelfel R, Gohlke F. The subacromial impingement syndrome of the shoulder treated by conventional physiotherapy, self-training, and a shoulder brace: results of a prospective, randomized study. Journal of Shoulder and Elbow Surgery. 2004 Jul $1 ; 13(4): 417-23$

[47] Wang CJ, Huang HY, Pai CH. Shock wave-enhanced neovascularization at the tendon-bone junction: an experiment in dogs. The journal of foot and ankle surgery. 2002 Jan 1;41(1):16-22.

[48] Yiasemides R, Halaki M, Cathers I, Ginn KA. Does passive mobilization of shoulder region joints provide additional benefit over advice and exercise alone for people who have shoulder pain and minimal movement restriction? A randomized controlled trial. Physical therapy. 2011 Feb $1 ; 91(2): 178-89$.

[49] Alper Kartal, Esin Ergin, "Investigation of the Effect of 6-week Yoga Exercises on Balance, Flexibility, and Strength in Soccer Players," International Journal of Human Movement and Sports Sciences, Vol. 8, No. 3, pp. 91 - 96, 2020. DOI: $10.13189 /$ saj.2020.080303.

[50] Yustinus Sukarmin, Japhet Ndayisenga, "Evaluation of Burundi Physical Education Teachers, Coaches, and Athletes' Sport Nutrition, Massage, and Physiotherapeutic Exercises Knowledge," International Journal of Human Movement and Sports Sciences, Vol. 8, No. 4, pp. 154 - 159, 2020. DOI: $10.13189 /$ saj.2020.080408.

[51] Ridho Bahtra, Moch. Asmawi, Widiastuti, Firmansyah Dlis, "Improved $\mathrm{VO}_{2 \mathrm{Max}}$ : The Effectiveness of Basic Soccer Training at a Young Age," International Journal of Human Movement and Sports Sciences, Vol. 8, No. 3, pp. 97 - 102, 2020. DOI: $10.13189 /$ saj.2020.080304 
[52] Gasibat Q, Simbak NB, Aziz AA, Petridis L, Tróznai Z. Stretching exercises to prevent work-related musculoskeletal disorders: A review article. AJSSM. 2017 Aug;5(2):27-37.
[53] Moflehi D, Kok LY, Tengku-Fadilah TK, Amri S. Effect of single-session aerobic exercise with varying intensities on lipid peroxidation and muscle-damage markers in sedentary males. Global journal of health science. 2012 Jul;4(4):48. 